\title{
Analysis of Surface Relief Evolution of Polycrystalline Aluminum Alloy Under Static Deformation
}

\author{
Pavlo Maruschak ${ }^{1, \text { a) }}$, Igor Konovalenko², Sergey Panin ${ }^{2,3)}$, \\ and Roman Bishchak ${ }^{4}$ \\ ${ }^{1}$ Ternopil National Ivan Pul'uj Technical University, Ternopil, 46001, Ukraine \\ ${ }^{2}$ Institute of Strength Physics and Materials Science SB RAS, Tomsk, 634055, Russia \\ ${ }^{3}$ National Research Tomsk Polytechnic University, Tomsk, 634050, Russia \\ ${ }^{4}$ Ivano-Frankivsk National Technical University of Oil and Gas, Ivano-Frankivsk, 76018, Ukraine \\ a) Corresponding author: maruschak.tu.edu.te.ua
}

\begin{abstract}
The peculiarities of using the surface as a damage sensor of the A2024 aluminum alloy under significant plastic strains are analyzed. Physical and mechanical regularities in the spatial self-organization of the structural and mechanical defects are found, and their relationship with the pore formation and deformation processes inside the material is established. It is proved that the surface relief formation can be used as the engineering parameter of the A2024 aluminum alloy damage under static deformation.
\end{abstract}

Keywords: strength, deformation, fracture, surface relief, mesomechanics

\section{INTRODUCTION}

One of the well-known approaches to obtaining information about the loading and damage history of structures is using the strain gauges of integral type (SGIT) [1]. They are bonded directly to the object under control, the gauge being able to "sense" the whole spectrum of loads, which affect the condition of its surface. Surface defects of SGITs are the initial information for evaluating the technical condition of the object analyzed $[2,3]$. There is a number of spheres, where SGITs can be used to diagnose the state of relevant structures, in particular: main gas- and oil pipelines, bridges, civil and military aircrafts. As a rule, the sensitive element of the gauge is made from either monocrystalline or polycrystalline aluminum $[4,5]$. However, in order to determine the damage parameters of the aluminum alloy basing on the deformation relief, it is necessary to find and investigate regularities in the variation of these parameters depending on the material loading conditions. In this case, the multilevel approach to the investigation of behavior of the loaded hard bodies is very important, because it allows obtaining the generalized picture of the material behavior at different structural levels, as suggested by physical mesomechanics [6].

This work investigates the influence of the macroscopic stress state type on the nature of localization of plastic yielding on the A2024 aluminum alloy surface, using the polycrystalline aluminum alloy as an example. The macroscopic stress state is preconditioned by the method of loading and geometrical peculiarities of specimens.

\section{RESEARCH TECHNIQUE}

The choice of the A2024 aluminum alloy for the investigation is preconditioned by the need of investigating the kinetics of damage accumulation in the aircraft material under static tensioning. This is important, since A2024 aluminum can be used as the fatigue accumulation sensor in aircraft. The investigation was carried out using the updated $\mathrm{ZD}-100 \mathrm{Pu}$ hydraulic static testing machine, the specimen strain being recorded using a strain gauge. The surface of specimens was photographed at different values of relative strain. The experimental investigation of the

(C) 2014 AIP Publishing LLC 978-0-7354-1260-6/\$30.00 
damage parameter evolution under static loading was performed, which allowed tracing the processes of the deformation relief development under tensile stresses, as well as making quantitative assessment of sensitivity of the A2024 aluminum alloy deformation relief evolution, and systematizing the relief development processes.

\section{ALGORITHM FOR PROCESSING IMAGES}

The surface relief parameters of the polycrystalline aluminum fatigue sensor were calculated by analyzing the photo images of the specimen surface $I_{0}(x, y)$ (Fig. 1(a)). The algorithm consists of two stages [5, 7]. The first (preliminary) stage includes the following operations: illumination equalization, filtering, and binarization. The second stage included the analysis of the obtained image and the calculation of the quantitative integral characteristics, which allow evaluation of the general condition of the specimen [8]. Due to the non-uniform illumination of different zones, the initial image of the surface (from Fig. 1(a) to Fig. 1(d)) has a partially distorted picture of the distribution of surface formations. The illumination equalization was performed in order to eliminate this deficiency. To this end, the image was folded using the low-frequency filter, and the low-frequency component was further removed from the initial image. With a view to obtaining the approximate image of illumination $l(x, y)$, the Gaussian filter with a large kernel size was used. The image with the equalized illumination was obtained as follows:

$$
I_{L}(x, y)=K_{L} \frac{I_{0}(x, y)}{l^{\prime}(x, y)}
$$

where $K_{L}=\max \left(l^{\prime}(x, y)\right)$ is the illumination equalization coefficient.

The next step of the algorithm - binary transformation - is intended for the preliminary image distribution into two clusters [5]: background and object sought. In image $I_{B}$ obtained, white pixels correspond to the background, and black pixels correspond to the recognized surface formations (Fig. 1(f-j)). The surface damage coefficient was used as the integral parameter for evaluating the condition of the surface analyzed:

$$
F=\frac{N_{b}}{N} \cdot 100 \%=\frac{N_{b}}{m n} \cdot 100 \%,
$$

where $N_{b}$ is the number of image pixels, which belong to the cluster of the objects recognized; $N$ is the general number of image pixels.

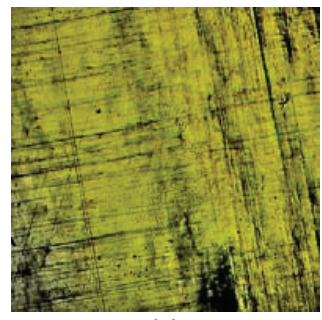

(a)

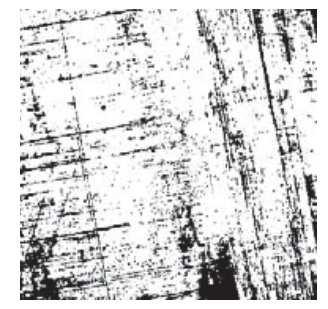

(e)

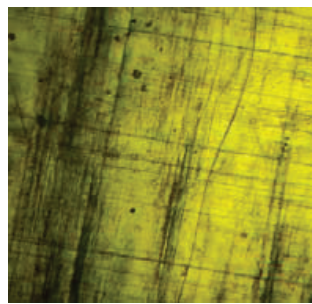

(b)

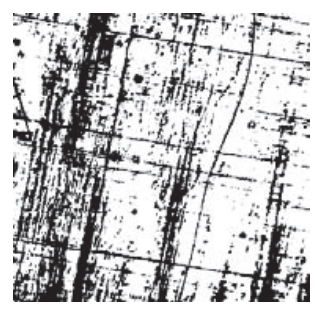

(f)

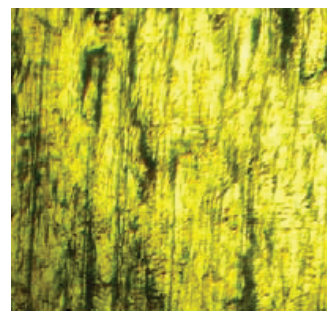

(c)

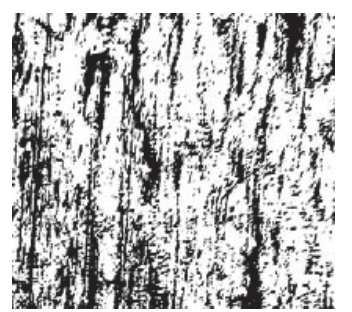

(g)

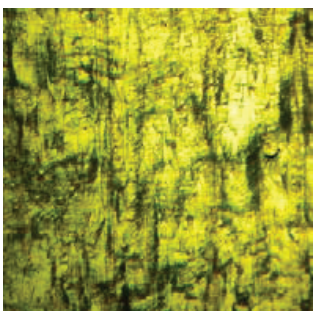

(d)

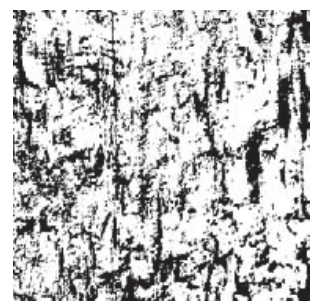

(h)

FIGURE 1. Initial images of analyzed surface at unloading points of complete diagram (Table 1) (a-d); and images that illustrate result of clusterization $(\mathrm{e}-\mathrm{h})$ (white pixels - background, black pixels - deformation relief). $\varepsilon=0(\mathrm{a}, \mathrm{e}), \varepsilon=2.6 \%(\mathrm{~b}, \mathrm{f}), 13 \%(\mathrm{c}, \mathrm{g}), 15.5 \%(\mathrm{~d}, \mathrm{~h})$ 
TABLE 1. Numeric results of analysis of deformed A2024 aluminum alloy specimen

\begin{tabular}{lccc}
\hline \multicolumn{1}{c}{ Parameter Type } & \multicolumn{3}{c}{ Parameter Value, $\%$} \\
\hline Longitudinal deformation $\varepsilon$ & 2.6 & 13.0 & 15.5 and failure \\
\hline Relative area of deformation relief $F$ & 17 & 27 & 33 \\
\hline
\end{tabular}

Let us consider the condition of the analyzed image surface from the viewpoint of the graded nature of the defect accumulation. The main stages of the software include transformation of the digital image of the surface into the black-and-white one; distinguishing individual clusters of the deformation relief; distinguishing contours of the deformation relief clusters, etc. (Fig. 1).

1. The manifestations of micro-yielding mostly of dislocation type are found in Fig. 1(a). Distortions of the grid and plastic deformation take place in separate local zones of the material surface layer.

2. At this stage, the formation of individual clear-cut deformation bands is possible in the local sections of the specimen (Fig. 1(b)).

3. The stage of macro-yielding is accompanied by the active plastic deformation throughout the volume of the material surface layer. The generation of plastic shears leads to a significant increase in the area of deformed sections (Fig. 1(c, d)).

4. It is quite hard to separate the final stage for A2024 aluminum alloy (as well as for many other materials without a clear-cut transition from macro-deformation to failure) from the stage of failure.

The analysis of optical images of the deformation relief was performed using the specially developed software, which allowed determining the deformation relief parameters (Table 1).

Figure 2 shows the failure micromechanisms of the A2024 aluminum alloy. Although the surface of the quasistatic failure is covered with the separation dimples, they no longer form the ductile surface of failure, but are ductile-brittle by nature (Fig. 2(a)).

The fracture surface is formed by cavities and facets with clear-cut boundaries (Fig. 2(b)). The secondary microcracks located near the cavities and on the sides of the facets, and local sections of quasi-spalling in the vicinity of the secondary phase particles can be seen very clearly (Fig. 2(c)). Such surface morphology can testify the fact that failure occurred due to the separation along the intra-crystalline areas in the ductile-brittle manner. The cracks that appeared in facets are the result of local plastic deformations at the preliminary stage.

\section{DISCUSSION AND GENERALIZATION}

The area of damage allows diagnosing the structural state of the material and, consequently, its properties and residual life. The obtained results supplement the description of the deformation behavior of the material at the macrolevel, which is derived from the stress-strain curves, i.e. they reproduce the evolution of the structure with defects. The deformation relief evolution under static tensioning allows considering it as an indicator of the accumulated deformation damage. A peculiarity of the internal structure of the surface layer within the deformation relief formation zone is the simultaneous presence of a number of dislocation structures, including the chaotic distributions of dislocations, band formations, long dislocation accumulations [2]. Three characteristic scale levels of deformation and failure of materials are analyzed. The possibilities of using different indicators for the evaluation of the aircraft structure material degradation are established. To this end, it is proposed to use a complex of methods, which allow detecting it at the micro-, meso- and macrolevels (see Table 2).

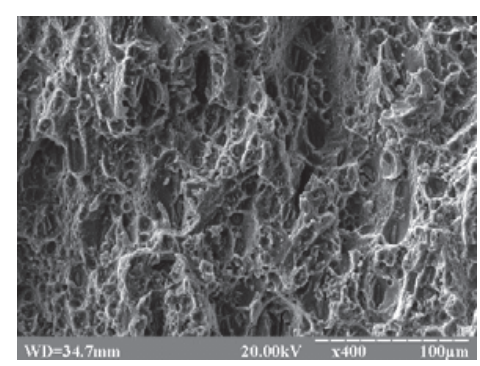

(a)

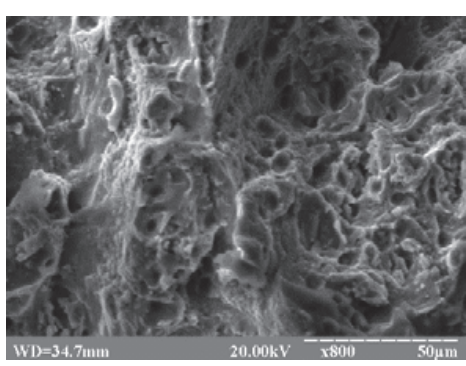

(b)

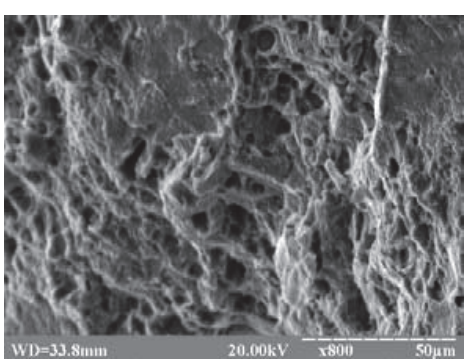

(c)

FIGURE 2. Micromechanisms of static failure of A2024 aluminum alloy 
TABLE 2. Damage evaluation methods and structural levels of deformation

\begin{tabular}{lccc}
\hline \multirow{2}{*}{$\begin{array}{c}\text { Parameters and Approaches of the Deformation } \\
\text { Process Analysis }\end{array}$} & \multicolumn{2}{c}{ Structural Levels of Material Damage } \\
\cline { 2 - 4 } & Micro & Meso & Macro \\
\hline Optical-digital analysis & - & + & + \\
\hline Strain measurements & - & - & + \\
\hline Fractodiagnostics & + & - & + \\
\hline
\end{tabular}

\section{SUMMARY}

The developed method is based on the computer-aided optical control of the surface condition of the A2024 aluminum alloy. The specimen surface is shown to be a stress concentrator at meso- and macro - levels. Using the example of polycrystalline structures, it was shown that the special role of the surface is connected with the kinetics of plastic yielding, i.e. with the appearance of the first plastic shears and strain localization on the surface of the deformed material. The computer-aided optical-digital diagnostics of the A2024 aluminum alloy surface is based on the analysis of the surface deformation relief. It is one of the promising damage assessment methods for aluminum alloys that are used for the production of fuselage shell of civil aircrafts.

\section{REFERENCES}

1. V. N. Syzrantsev and S. L. Golofast, Measurements of Cyclic Strains and Prediction of the Lifetime of Parts Based on Integral Strain Sensor Readings (Nauka, Novosibirsk, 2004).

2. M. Petrasek, S. Ignatovich, M. Karuskevich, and T. Maslak, Adv. Military Technol. 8, 833-91 (2013).

3. S. R. Ignatovich, A. Menou, M. V. Karuskevich, and P. O. Maruschak, Theor. Appl. Fract. Mech. 65, 23 (2013).

4. S. V. Panin, Yu. A. Altukhov, P. S. Lyubutin, A. V. Byakov, and S. A. Khizhnyak, Optoel. Instr. Data Proc. 49, 234 (2013).

5. I. Konovalenko, P. Maruschak, A. Menou, et al., "A novel algorithm for damage analysis of fatigue sensor by surface deformation relief parameters", in Proc. Int. Symposium on Operational Research and Applications, Morocco, Marrakech, 2013, pp. 678-684.

6. V.E. Panin, Theor. Appl. Fract. Mech. 37, 261 (2001).

7. I. Konovalenko and P. Maruschak, "Investigation of cracked surface condition using digital image analysis", in Proc. 12 Int. Conf. "The Experience of Designing and Application of CAD Systems in Microelectronics", Polyana-Svalyava, 2013, p. 178.

8. P. Yasniy, P. Maruschak, and I. Konovalenko, Strain 47, 238 (2011). 